\title{
Éclosion prolongée de coqueluche en Ontario ayant pour origine une communauté religieuse sous-immunisée
} Deeks $\mathbf{S L}^{1,2^{*}}$, Lim $\mathbf{G H}^{1}$, Walton $\mathbf{R}^{1}$, Fediurek $\mathbf{J}^{1}$, Lam $\mathbf{F}^{1}$, Walker $\mathbf{C}^{3}$, Walters $\mathbf{J}^{4}$ et Crowcroft $\mathbf{N} \mathbf{S}^{1,2,5}$

1 Santé publique Ontario, Toronto (ON

2 Dalla Lana School of Public Health, Université de Toronto, Toronto (ON)

3 Circonscription sanitaire Elgin-St. Thomas

4 Circonscription sanitaire de Wellington-Dufferin-Guelph

5 Département de médecine laboratoire et biopathologie, faculté de médecine, Université de Toronto, Toronto (ON)

* Auteure-ressource : shelley.deeks@oahpp.ca

\section{Résumé}

Contexte : En Ontario, une éclosion prolongée de coqueluche s'est déclarée en novembre 2011 dans une communauté religieuse sous-immunisée, pour ensuite se propager dans la population générale et dans une deuxième communauté religieuse de la même région.

Objectif : À comparer l'épidémiologie de la coqueluche dans les communautés religieuses avec celle dans la population générale des administrations touchées.

Méthodes : L'analyse porte sur les cas signalés par l'intermédiaire du Système d'information sur la santé publique intégré (SISP-i) entre le 1er novembre 2011 et le 15 avril 2013 qui répondaient à la définition de cas liés à l'éclosion. Le personnel des bureaux de santé publique a effectué des investigations de cas pour déterminer si les cas étaient des membres de communautés religieuses et a recueilli des renseignements sur l'état immunitaire, le traitement et les résultats.

Résultats : Au total, 443 cas confirmés et probables liés à une éclosion ont été signalés dans 7 circonscriptions sanitaires. L'éclosion s'est déclarée dans une communauté religieuse (138 cas) avant de se propager dans la population générale de la région (273 cas). De plus, 32 cas ont été signalés dans une deuxième communauté religieuse sous-immunisée de la région. Au total, 13 cas ont été hospitalisés, et aucun décès n'a été déclaré. La communauté religieuse a connu une flambée d'activité de la maladie plus tôt; les cas étaient beaucoup plus jeunes, plus susceptibles de présenter un risque élevé de contracter la coqueluche et plus susceptibles d'être sous-immunisés. Dans la population générale complètement immunisée, $51 \%$ des cas étaient âgés de 10 à 14 ans et leur dernière immunisation remontait à 5,6 ans (nombre d'années médian).

Conclusion : L'épidémiologie de la coqueluche dans la communauté sous-immunisée est différente de celle dans la population générale. Pendant une éclosion, on doit s'attendre à ce que la coqueluche se transmette à la population générale; toutefois, compte tenu de la proportion des cas dont l'immunisation est à jour, une étude plus poussée s'avère nécessaire.

\section{Introduction}

Entre 2010 et 2012, des éclosions de coqueluche se sont déclarées dans un certain nombre d'administrations en Amérique du Nord ${ }^{1-5}$. La coqueluche est une infection des voies respiratoires très contagieuse causée par Bordetella pertussis, qui est dangereuse surtout pour les nourrissons de moins d'un an, particulièrement chez ceux de moins de quatre mois ${ }^{6,7}$. La lutte contre la maladie s'avère difficile, car la période de transmissibilité est longue, le tableau clinique de la coqueluche est atypique chez les enfants immunisés et les adultes et la maladie peut être difficile à diagnostiquer ${ }^{8,9,10}$. 
En Ontario, les vaccins contre la coqueluche sont offerts depuis 1943. En 1984, un vaccin anticoquelucheux adsorbé à germes entiers combiné était utilisé dans le cadre du programme d'immunisation public universel de la province. En 1997, le vaccin acellulaire contre la coqueluche remplace le vaccin à germes entiers et est administré sous forme de vaccin combiné (diphtérie [D], tétanos [T], coqueluche acellulaire [Ca], vaccin antipoliomyélitique inactivé [VPI] et Haemophilus influenzae de type b [Hib]) à 2, 4 et 6 mois. On administre ensuite une dose de rappel du vaccin pentavalent (DCaT-VPI-Hib) à 18 mois et une dose de rappel du vaccin quadrivalent (DCaT-VPI) aux enfants de 4 à 6 ans. Une dose pour adolescent a été financée par l'État en 2003, soit le vaccin DCaT donné aux adolescents âgés de 14 à 16 ans. Enfin, en 2011, un programme d'immunisation des adultes d'une dose unique du vaccin contre la coqueluche a été lancé ${ }^{11}$.

Le 25 janvier 2012, une éclosion de coqueluche s'est déclarée dans la province après qu'une grappe de cas a été détectée au sein d'une communauté religieuse sous-immunisée (communauté religieuse A) dans le sud-ouest de I'Ontario, au Canada. Au départ, l'éclosion se limitait à trois circonscriptions sanitaires ainsi qu'à une communauté religieuse $(A)$, mais elle s'est ensuite propagée dans la population générale ainsi que dans une deuxième communauté religieuse sous-immunisée $(B)$, touchant sept circonscriptions sanitaires de la province.

Notre objectif est de faire rapport de notre enquête sur cette éclosion au moyen d'une comparaison de l'épidémiologie dans les communautés religieuses avec l'épidémiologie dans la population générale fondée sur les données démographiques, l'état immunitaire, le traitement et les résultats.

\section{Méthodes}

\section{Surveillance}

Selon la loi de l'Ontario, la coqueluche est une maladie à déclaration obligatoire (population de la province : 13,5 millions en 2012) ${ }^{12}$. Les cas sont décelés grâce à une surveillance passive et les renseignements sur les cas sont consignés dans un registre provincial des maladies à déclaration obligatoire, soit le Système d'information sur la santé publique intégré (SISP-i). Les définitions de cas de coqueluche liés à l'éclosion, qui comprenaient une dimension géographique, ont été établies, puis modifiées à trois reprises au cours de l'éclosion afin de tenir compte de l'évolution de la situation. La période de l'éclosion était du 1er novembre 2011 au 15 avril 2013. Le 22 août 2013, nous avons extrait les données du SISP-i qui répondaient aux définitions de cas de coqueluche confirmés et probables liés à l'éclosion. Les données extraites comptaient des données démographiques, des résultats de laboratoire, des renseignements sur les hospitalisations, des résultats et des antécédents de vaccination, mais leur intégralité variait selon les champs. Nous avons déterminé la date de l'épisode de chaque cas à l'aide d'une hiérarchie normalisée : la date d'apparition des symptômes était privilégiée, sinon la date de prélèvement des échantillons était sélectionnée ou la date de déclaration du cas était utilisée si les deux autres dates n'étaient pas indiquées.

Au début de l'éclosion, en complément des données du SISP-i, nous avons recueilli des données supplémentaires tirées des notes sur les cas à l'aide d'un formulaire amélioré de déclaration des cas afin d'obtenir de l'information sur le degré de risque que présentaient les cas (élevé ou non) ainsi que sur les comportements adoptés par rapport à la santé et le traitement. Un examen manuel des déclarations de cas a été réalisé pour les cas signalés entre le 1er novembre 2011 et le 15 août 2012. Les personnes que nous avons classées comme présentant un risque élevé de coqueluche étaient les femmes enceintes et les nourrissons de moins d'un an. Nous avons également vérifié, pour chaque cas, si des tests de dépistage en laboratoire avaient été effectués, si une antibiothérapie avait été entreprise et quel était l'intervalle avant la recherche de traitement.

Les bureaux de santé publique devaient déterminer les liens avec les communautés religieuses sous-immunisées dans le SISP-i. Nous avons utilisé des identifiants précis d'exposition pour déterminer si les cas faisaient partie de l'une des deux communautés religieuses sous-immunisées, soit $A$ et $B$. Pour certaines analyses, les communautés religieuses $A$ et $B$ ont été combinées. Nous avons supposé que les cas qui n'avaient pas été associés à un identifiant d'exposition provenaient du reste de la population des circonscriptions sanitaires (population générale). 
Comme l'évaluation de l'état immunitaire à partir d'un seul champ du SISP-i posait problème, nous avons examiné les champs suivants pour tous les cas : agent immunisant, dates d'administration des vaccins, vaccination pertinente à jour et facteur de risque. Le nombre de doses du vaccin anticoquelucheux reçues a été établi selon le nombre de dates d'administration déclaré pour chaque cas. Nous avons déterminé la validité des doses en considérant les exigences relatives à l'âge minimal et à l'intervalle minimal entre les doses, selon le calendrier financé par l'État en Ontario ${ }^{13}$. Pour qu'une dose soit considérée comme valide, l'intervalle avant l'apparition de la maladie devait être d'au moins 14 jours. Nous avons défini les cas qui n'avaient aucune dose valide (c.-à-d. aucune date d'administration) comme non immunisés s'ils ne présentaient aucune vaccination pertinente ou si leur facteur de risque était non vacciné; sinon, nous les avons définis comme ayant un état immunitaire inconnu. Nous avons défini les cas ayant reçu au moins une dose valide comme complet pour l'âge (c.-à-d. à jour), conformément au calendrier d'immunisation provincial et aux lignes directrices établies par le Comité consultatif national de l'immunisation ${ }^{11,14}$.

\section{Analyses statistiques}

Nous avons calculé les taux d'incidence pour 100000 habitants à l'aide des données démographiques de Statistique Canada obtenues par l'intermédiaire d'IntelliHealth Ontario. Comme les données de 2013 sur la population selon la circonscription sanitaire n'étaient pas disponibles au moment de l'analyse, nous avons utilisé celles de 2012. Des analyses descriptives et des tests statistiques ont été effectués avec la version 19 du SPSS (IBM, Armonk, NY, É.-U.) et de la version 9.2 du SAS. Nous avons utilisé le test de Kruskal-Wallis pour comparer l'âge médian et les intervalles de temps pour certains résultats des différentes communautés; la signification statistique a été établie à $\mathrm{p}<0,05$. Nous avons exclu, au besoin, les données manquantes des analyses.

\section{Résultats}

\section{Épidémiologie descriptive}

Au total, 443 cas de coqueluche ont été signalés dans 7 circonscriptions sanitaires touchées au cours de la période d'éclosion. Parmi ces cas, $344(77,7 \%)$ ont été classés comme confirmés d'après la confirmation de la maladie en laboratoire ou un lien épidémiologique avec un cas confirmé en laboratoire. Les 99 cas restants ont été classés comme probables d'après les symptômes. L'âge des personnes infectées variait entre 14 jours et 68 ans, l'âge médian étant de 9,4 ans (données sur l'âge manquantes pour 6 cas). Les femmes représentaient $56 \%$ des cas. Le taux d'incidence annualisé pendant l'éclosion était de 25,7 cas pour 100000 habitants (en 2012, la population des 7 circonscriptions sanitaires était de 1,2 million de personnes). Durant la même période, le taux provincial global était de 6,1 cas de coqueluche pour 100000 habitants. En tout, 13 cas (2,9\%) ont été hospitalisés, et aucun décès n'a été signalé. Tous les cas hospitalisés étaient des enfants, dont huit étaient âgés de moins d'un an.

Sur les 443 cas, $31,2 \%$ provenaient de la communauté religieuse $A ; 7,2 \%$, de la communauté religieuse $B$ et $61,6 \%$, de la population générale (Tableau 1). La répartition des cas selon l'âge dans les deux communautés religieuses était différente de celle dans la population générale (Figure 1). L'âge médian des cas de la population générale était d'environ 10 ans, ce qui était beaucoup plus élevé que l'âge médian des cas des deux communautés religieuses $(p<0,001)$. Bien que plus de la moitié des cas déclarés dans les communautés religieuses $A$ et $B$ étaient âgés de moins de 5 ans, seulement $19,8 \%$ des cas de la population générale ont été déclarés chez les enfants de ce groupe d'âge $(p<0,0001)$. Dans la population générale, la proportion de cas la plus importante $(28,2 \%)$ était dans le groupe d'âge de 10 à 14 ans. Les probabilités d'hospitalisation ne variaient pas selon la communauté. 
Tableau 1 : Comparaison de résultats sélectionnés parmi les cas des communautés religieuses $A$ et $B$ et de la population générale à partir des données du SISP-i et des formulaires de déclaration de cas

\begin{tabular}{|c|c|c|c|}
\hline & Communauté religieuse $\mathbf{A}$ & Population générale & Communauté religieuse B \\
\hline \multicolumn{4}{|l|}{ Données du SISP-i $(n=443)$} \\
\hline Nombre de cas (\%) & $138(31,2 \%)$ & $273(61,6 \%)$ & $32(7,2 \%)$ \\
\hline Âge médian (années) & 3,7 & 13,2 & 3,9 \\
\hline $\begin{array}{l}\text { Proportion d'enfants } \\
\text { de moins de } 5 \text { ans }\end{array}$ & $60,6 \%$ & $19,8 \%$ & $59,4 \%$ \\
\hline \multicolumn{4}{|c|}{ Données des formulaires de déclaration de cas $(n=303)$} \\
\hline Nombre de formulaires remplis & 115 & 188 & 0 \\
\hline \% risque élevé & $22,6 \%$ & $5,3 \%$ & S.O. \\
\hline$\%$ tests de laboratoire & $50,9 \%$ & $81,3 \%$ & S.O. \\
\hline
\end{tabular}

S.O. = Sans objet

Figure 1 : Cas de coqueluche selon l'âge et le statut de la communauté : Ontario, du 1er novembre 2011 au 15 avril $2013\left(n=437^{*}\right)$

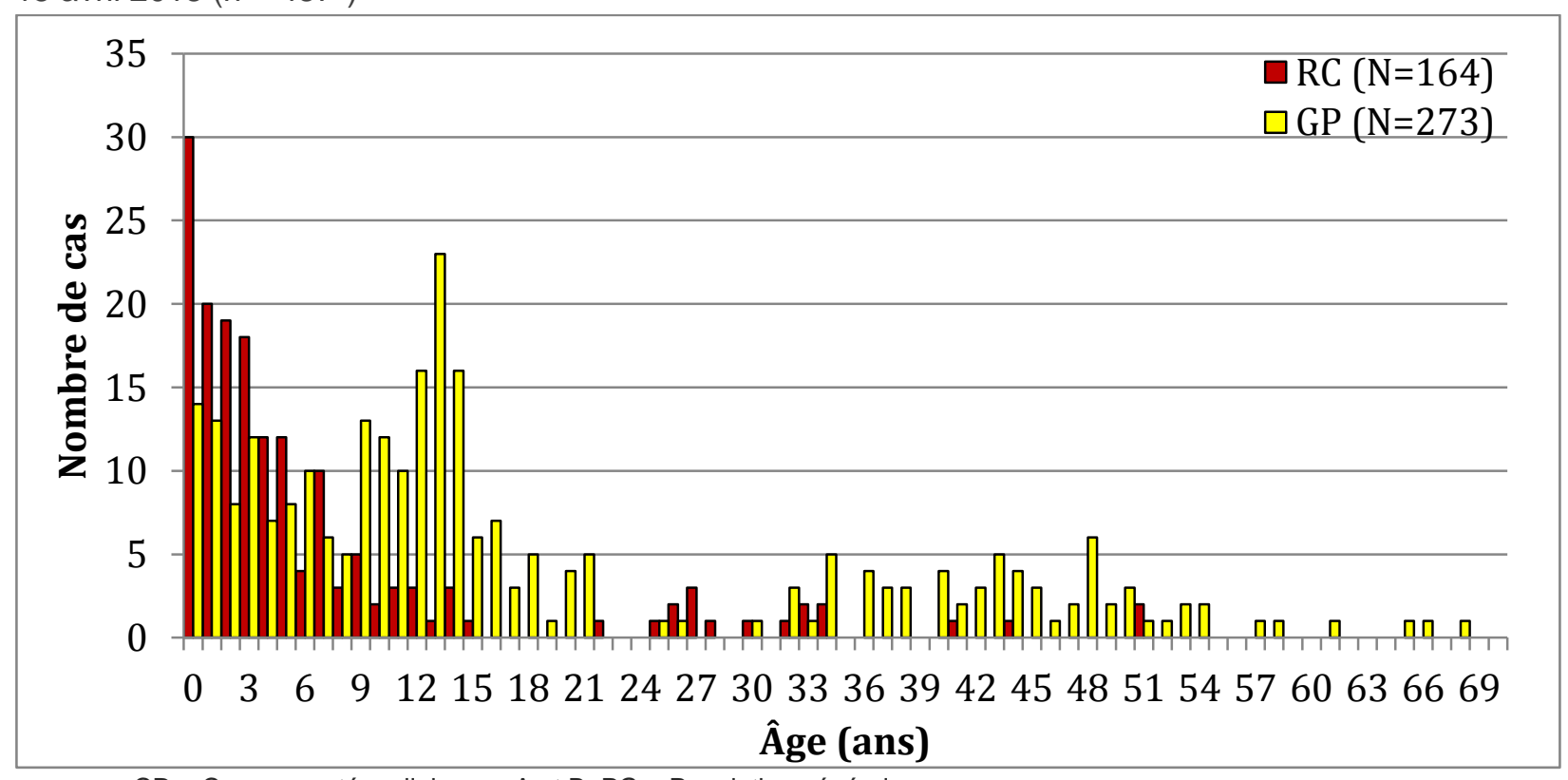

$\mathrm{CR}=$ Communautés religieuses $\mathrm{A}$ et $\mathrm{B} ; \mathrm{PG}=$ Population générale ${ }^{*}$ Exclut 6 cas dont l'âge est inconnu.

Nous avions accès à des formulaires améliorés de déclaration pour $68,4 \%$ des cas : 115 de la communauté religieuse $A$ et 188 de la population générale (aucun formulaire amélioré de déclaration de cas n'était disponible pour la communauté religieuse $\mathrm{B}$, car ces cas se sont déclarés plus tard au cours de l'éclosion). Le Tableau 1 indique que les cas présentant un risque élevé étaient plus nombreux dans la communauté religieuse $A$ que dans la population générale $(p<0,0001)$ et que les membres de la communauté religieuse $A$ étaient moins susceptibles de se soumettre au test de dépistage de la coqueluche en laboratoire recommandé $(p<0,0001)$ et d'entreprendre une antibiothérapie $(p<0,01)$. Comparativement aux cas de la population générale, les cas de 
cette communauté religieuse présentaient une plus longue période entre l'apparition des symptômes et le début du traitement $(p<0,005)$.

La répartition temporelle des cas était associée à une tendance bimodale durant l'éclosion (Figure 2). La première vague a atteint son sommet en janvier 2012 et se limitait presque exclusivement aux cas de la communauté religieuse $A$. En ce qui concerne la deuxième vague, le sommet a été atteint en juin 2012 et elle se composait principalement des cas de la population générale, même si la fin de la courbe épidémique était un mélange de cas, dont des cas de la communauté religieuse $B$.

Figure 2: Cas confirmés et probables liés à une éclosion $(n=443)$ selon la date d'apparition, la classification des cas et la communauté, dans le sud-ouest de l'Ontario (du 1er nov. 2011 au 15 avr. 2013)

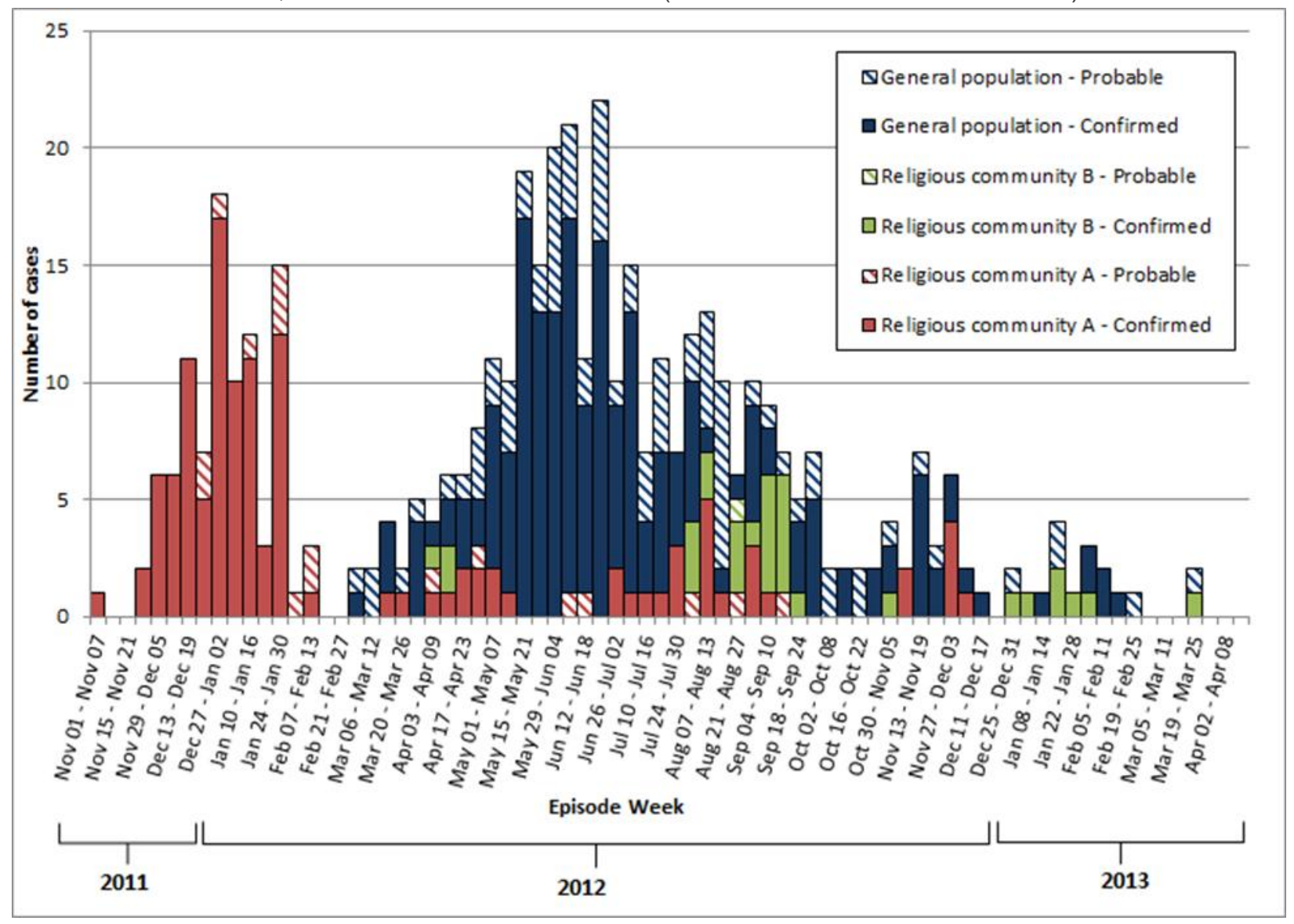

\section{Immunisation}

Nous avons été en mesure de déterminer l'état immunitaire de $85,1 \%(n=377)$ des cas. Parmi ceux-ci, $34,5 \%$ présentaient une immunisation complète pour l'âge; $11,9 \%$ étaient partiellement immunisés et $53,6 \%$ n'étaient pas immunisés. L'état immunitaire était semblable entre les deux communautés religieuses, ce qui nous a permis de combiner leurs données aux fins d'analyse. Nous avons constaté une différence significative entre les communautés religieuses et la population générale relativement à la proportion de cas immunisés et complètement immunisés pour leur âge $(p<0,01)$. Dans les deux communautés religieuses, $86,6 \%$ des cas n'étaient pas immunisés (état immunitaire connu) par rapport à seulement 32,0\% dans la population générale. La répartition selon l'âge des cas non immunisés était distincte pour chaque communauté. Les cas non immunisés dans les communautés religieuses étaient plus jeunes que ceux déclarés dans la population générale (âge médian de 3,0 par rapport à 8,4 ans, respectivement, $p<0,0001)$. En revanche, 6,0\% $(n=9)$ des cas dont l'état immunitaire était connu étaient complètement immunisés pour leur âge, comparativement à $53,1 \%(n=121)$ dans la population générale $(p<0,0001)$. Dans la population générale, l'âge médian des cas complètement 
immunisés pour leur âge était de 11,7 ans (plage : de 2 mois à 43 ans), et plus de la moitié $(51,2 \%)$ des cas étaient âgés de 10 à 14 ans. Le nombre d'années médian depuis la dernière immunisation était de 5,6 ans (plage : de 15 jours à 9,9 ans).

\section{Analyse}

L'Ontario a connu une éclosion de coqueluche qui a duré plus de 17 mois. Nous avons constaté que l'épidémiologie de la coqueluche dans la communauté sous-immunisée était différente de celle dans la population générale : la répartition selon l'âge était plus jeune, la proportion des cas non immunisée était plus importante et la proportion des cas présentant un grand risque était plus élevée au sein de la communauté. L'épidémiologie de la coqueluche dans la communauté sous-immunisée correspond davantage à celle observée dans les pays moins industrialisés et est semblable à celle que l'on observait en Amérique du Nord avant la mise en œuvre des programmes d'immunisation ${ }^{15,16}$. Malgré le fait que les personnes infectées étaient plus jeunes dans les communautés sous-immunisées, la maladie ne semblait pas plus grave. Même si $86,6 \%$ des cas de la communauté religieuse étaient sous-immunisés, certains membres étaient prêts à être vaccinés à l'occasion de certaines cliniques de sensibilisation organisées par les bureaux de santé publique, où des tests de dépistage et une chimioprophylaxie étaient également offerts.

Le personnel des bureaux de santé publique était préoccupé par le fait que certains membres des communautés religieuses refusaient le suivi après le test de confirmation en laboratoire. II était tout de même rassurant de savoir que près de la moitié des cas se soumettaient aux tests, même si les cas de la communauté religieuse étaient moins nombreux que ceux de la population générale à subir des tests en laboratoire. De plus, il était encourageant d'observer que la majorité des personnes de tous les milieux étaient traitées une fois le cas confirmé, bien que les membres des communautés religieuses aient été moins nombreux à le faire. Le délai avant le traitement chez les membres de la communauté religieuse était potentiellement attribuable aux interventions de la santé publique.

On peut s'attendre à ce que la coqueluche se transmette à la population générale pendant une éclosion, plus précisément en raison de l'infectiosité de l'organisme. Comme l'Ontario ne dispose pas de registre d'immunisation complet, nous n'avons pas été en mesure de déterminer la couverture vaccinale de la population générale ni l'efficacité du vaccin. Le manque de données utilisées en guise de dénominateur concernant les communautés religieuses constitue également un obstacle pour ce qui est d'évaluer l'efficacité du vaccin au moyen de la méthode de dépistage. Cependant, le système d'information sur l'immunisation en milieu scolaire de l'Ontario a permis de déterminer, pour l'année scolaire 2011-2012, que la proportion des élèves de 7 et de 17 ans de la province dont l'immunisation contre la coqueluche était à jour était de $76,0 \%$ et de $67,7 \%$, respectivement ${ }^{17}$. Or, cette couverture ne suffit pas pour empêcher la transmission de la coqueluche au sein des communautés, plus particulièrement dans les régions où la couverture est moindre en raison d'objections religieuse ou de conscience à la vaccination.

Pendant cette éclosion, la proportion des cas de la population générale qui n'étaient pas complètement immunisés était inquiétante, tout comme lors d'autres éclosions récentes en Amérique du Nord ${ }^{1,4}$. En Ontario, le vaccin acellulaire contre la coqueluche a été introduit en 1997, de sorte que la première cohorte d'enfants complètement immunisés par le vaccin acellulaire seulement devait être âgée d'environ 14 ou 15 ans pendant cette éclosion. Les enfants de 10 à 14 ans représentent 5,7\% de la population de l'Ontario, mais pesait pour 20,4 $\%$ de l'ensemble des cas signalés pendant l'éclosion, pour $28,2 \%$ des cas signalés dans la population générale et pour $51,2 \%$ des cas de la population générale dont l'immunisation était à jour. Ces données justifient une étude plus approfondie et semblent indiquer une diminution de l'immunité associée au vaccin acellulaire contre la coqueluche, ce qui a également été observé aux États-Unis ${ }^{1,18,19}$.

Notre analyse présente certaines limites. II est difficile d'évaluer avec exactitude l'état immunitaire à partir des données du SISP-i pour des maladies comme la coqueluche qui présentent des calendriers d'immunisation complexes. De plus, les dossiers ne contenaient pas toujours tous les renseignements cliniques, et l'état immunitaire était inconnu chez $14,9 \%$ des cas, malgré le fait que le personnel des bureaux de santé publique ait effectué un suivi important. Nous avons pu compenser ces limites dans une certaine mesure grâce à l'utilisation 
des formulaires améliorés de déclaration des cas remplis au début de l'éclosion. Étant donné la longue durée de l'éclosion et les ressources humaines requises pour remplir ces formulaires, ces derniers n'ont été employés que pour les cas qui se sont déclarés au cours des neuf premiers mois et demi de l'éclosion, ce qui peut avoir une incidence sur leur représentativité. Enfin, comme il a déjà été mentionné, nous n'avons pas été en mesure de calculer l'efficacité du vaccin en raison de l'absence d'un registre d'immunisation provincial complet.

\section{Conclusion}

Cette éclosion a permis de comparer l'épidémiologie de la coqueluche dans diverses communautés au sein d'une même région géographique. On peut s'attendre à ce que la coqueluche se transmette de communautés sousimmunisées à la population générale; toutefois, le nombre de cas d'adolescents dont l'immunisation était à jour semble indiquer une diminution de l'immunité conférée par le vaccin.

\section{Références}

1. Klein N.P., Bartlett J., Fireman B. et al. «Comparative Effectiveness of Acellular Versus Whole-Cell Pertussis Vaccines in Teenagers ». Pediatrics. Juin 2013;131(6):e1716-22. doi: 10.1542/peds.2012-3836.

2. Pharmaceutical Services Division, Ministry of Health British Columbia. Pertussis Outbreak Management in Fraser Health and Vancouver Coastal Health Authorities.

http://www.health.gov.bc.ca/pharmacare/pdf/5d.pdf

3. Centers for Disease Control and Prevention. Pertussis Outbreak Trends. http://www.cdc.gov/pertussis/outbreaks/trends.html

4. Bureau du médecin-hygiéniste en chef, Nouveau-Brunswick, Canada. La coqueluche au Nouveau-Brunswick. http://www2.gnb.ca/content/gnb/fr/ministeres/bmhc/maladies_transmissibles/content/la_coqueluche.html

5. Centers for Disease Control and Prevention. Pertussis Outbreak Trends. http://www.cdc.gov/pertussis/outbreaks/trends.html.

6. von König C.H., Halperin S., Riffelmann M. et Guiso N. «Pertussis of adults and infants ». Lancet Infect Dis. 2 déc. 2002(12):744-50.

7. Guiso N. « Bordetella pertussis: Why is it still circulating? » J Infect. 5 oct. 2013. doi: pii: S01634453(13)00289-2. 10.1016/j.jinf.2013.09.022 [publié en ligne avant impression].

8. Deeks S., De Serres G., Boulianne N., Duval B., Rochette L., Dery P. et al. « Failure of physicians to consider the diagnosis of pertussis in children ». Clin Infect Dis. 1999;28(4):840-6.

9. Taylor Z.W., Ackerson B., Bronstein D.E., Lewis K., Steinberg E., Stone M.M., Viraraghavan R., Wong V.K. et Salzman M.B. « Wheezing in Children with Pertussis Associated with Delayed Pertussis Diagnosis ». Pediatr Infect Dis J. 28 oct. 2013. [publié en ligne avant impression].

10. Cornia P.B., Hersh A.L., Lipsky B.A., Newman TB, Gonzales R. « Does this coughing adolescent or adult patient have pertussis? 》 JAMA. 25 août 2010; 304(8):890-6. doi: 10.1001/jama.2010.1181.

11. Ministère de la Santé et des Soins de longue durée de l'Ontario. Calendriers de vaccination financée par le secteur public en Ontario - août 2011.

http://www.health.gov.on.ca/fr/public/programs/immunization/docs/schedule.pdf

12. Ministère de la Santé et des Soins de longue durée de l'Ontario. "Appendix B: Provincial Case Definitions for Reportable Disease: Pertussis ». Protocole concernant les maladies infectieuses, 2013.

http://www.health.gov.on.ca/fr/pro/programs/publichealth/oph_standards/infdispro.aspx

13. Ministère de la Santé et des Soins de longue durée de l'Ontario. Âges recommandés et intervalles minimums prescrits entre les doses de vaccins financés par les deniers publics et administrés systématiquement pendant l'enfance - août 2011 version 2.

http://www.health.gov.on.ca/en/pro/programs/immunization/docs/age_interval.pdf 
14. Comité consultatif national de l'immunisation (CCNI). Vaccin contre la coqueluche. Guide canadien d'immunisation. Édition évolutive. Ottawa : Agence de la santé publique du Canada; 2012. http://www.phac-aspc.gc.ca/publicat/cig-gci/p04-pert-coqu-fra.php

15. Gordon J.E. et Hood R.I. «Whooping cough and its epidemiological anomalies ». Am J Med Sci. Sept. $1951 ; 222(3): 333-61$.

16. Crowcroft N.S. et Pebody R.G. « Recent developments in pertussis ». Lancet. 10 juin 2006;367(9526): 1926-36.

17. Wilson S., Lim G.H., Fediurek J., McIntyre M.A. et Deeks S.L. 2011/12 Immunization coverage from Ontario, Canada: uptake and exemptions. National Foundation for Infectious Diseases' 16th Annual Conference on Vaccine Research, Baltimore, É.-U.-A., 22-24 avril 2013.[presentation orale]

18. Tartof S.Y., Lewis M., Kenyon C. et al. «Waning immunity to pertussis following 5 doses of DTaP ». Pediatrics. Avril 2013;131(4):e1047-52. doi: 10.1542/peds.2012-1928. Epub 11 mars 2013

19. Klein N.P., Bartlett J., Rowhani-Rahbar A., Fireman B. et Baxter R. « Waning protection after fifth dose of acellular pertussis vaccine in children ». N Engl J Med. 13 sept 2012;367(11):1012-9. doi: 10.1056/NEJMoa1200850.

\section{Remerciements}

Les auteurs tiennent à remercier toutes les personnes qui ont participé à l'intervention pendant l'éclosion des circonscriptions sanitaires touchées, du ministère de la Santé et des Soins de longue durée de l'Ontario et de Santé publique Ontario.

\section{Conflit d'intérêts}

Les auteurs n'ont aucun conflit d'intérêts à déclarer.

\section{Source de financement}

Aucun financement externe n'a été obtenu pour cette étude.

\section{Divulgation de la situation financière}

Les auteurs n'ont aucune relation financière à déclarer relativement à cet article. 